\title{
Comparison of Post-op quality of vision of spheric IOL and aspheric hydrophilic acrylic yellow tinted intraocular lens implantation following phacoemulsification cataract surgery
}

\begin{abstract}
Purpose: To compare the post-op visual quality of spheric and aspheric acrylic yellow tinted intraocular lens following phacoemulsification cataract surgery.

Place of study: Al-Majeed Laser Eye Hospital.

Methods: Total 60 subjects (aged 40-65years) with age related mature cataracts were included. Data was assembled by non-probability convenient sampling technique at $\mathrm{Al}$ Majeed Laser Eye Hospital. All patients presenting with mature cataract were include in this study without any other pathology and fundus abnormality. Pre and post-operative visual acuity was measured by using standard snellen visual acuity testing chart. For the purpose of accuracy repetitive values were taken for each patient. Post-operative vision was recorded one month followed by phacoemulsification. Comparisons was made between spheric and asphereic acrylic yellow tinted intraocular lens by applying (descriptive) statistics and paired sample $t$ test for the analysis of results.
\end{abstract}

Results: Quality of vision was compared post-operatively after implantation of aspheric hydrophilic acrylic IOL and spheric IOL in posterior chamber under the same phaco surgeon. All subjects after intra ocular implantation had better corrected visual acuity of $6 / 9$ and more improved $6 / 6$. There were found statistically differences in recorded visual acuity with aspheric yellow tinted iol implanation and spheric iol $(\mathrm{p}=0.15)$ between the two types of intra-ocular lens. The mean, standard deviation, t-value found with spheric IOL were.1867, .18889, 2.517 respectively. However, of aspheric hydrophilic acrylic intra-ocular lens mean, standard deviation and $\mathrm{t}$ values found $.800, .13493$ and 2.5493 respectively. There was significant variance in statistics and quality of vision between the two types of lenses.

Conclusions: This study concludes that post -operative improvement in quality of vision was better with aspheric hydrophilic yellow tinted lenses than spheric iol implantation. Implanting a foldable aspheric acrylic IOL gave much advantage in visual outcome to pseudophakes by protecting retina from light of shorter wavelength and decrease of chromatic aberration under the photopic circumstances particularly in subjects at hazard of age-related macular degeneration (AMD).
Volume II Issue 2 - 202 I

\author{
Safdar Hussain,' Asma Batool,,2 Saba Akram,'² \\ Hina manzoor, ${ }^{2}$ Ayesha Arshad ${ }^{3}$ \\ 'Consultant ophthalmologist at DHQ Dera ghazi khan, Pakistan \\ ${ }^{2}$ Department of optometry and vision sciences at The \\ University of Lahore, Pakistan \\ ${ }^{3}$ Optometrist at Bhawal Victoria hospital, Pakistan
}

Correspondence: Asma batool,Assistant professor, Department of optometry and vision sciences at The University of Lahore, Pakistan, Email Asmahusain02@gmail.com

Received: July 09, 202I | Published: August 06, 2021

\section{Introduction}

The latest surgical techniques not only give better cataract surgery but also provide good visual acuity due to the advancement in surgical techniques and invention of new design and materials of intraocular lens. Visual function enhancement and retinal protection against toxicity of light after the surgery of Cataract have become a matter of interest. ${ }^{1}$ Cataract is still the leading cause of blindness across the world. Even though the number of cataract operations has increased according to the result of WHO Vision 2020 initiative. After surgery poor results are the matter of importance and according to the population based studies in Asia, Africa and Latin America at least $20 \%$ of patients after cataract surgery have vision $<6 / 60$. The surgical method that would increase the vision after surgery will be a great contribution to Vision $2020 .^{2}$ The purpose of modern Cataract surgery includes minimal rate of Astigmatism after surgery, fast visual rehabilitation and the possible best uncorrected visual acuity. Couching to the latest micro-incision cataract surgical technique, passing through phases of the intra-capsular and the conventional extra-capsular method (ECCE) of cataract extraction, manual smallincision cataract surgery and phacoemulsification, the surgical cataract techniques have improved a lot. Micro-incision cataract surgical technique and conventional phacoemulsification are still practiced rarely across the world in developing countries including ours due to the high expense of machinery, expensive maintenance and difficulty in acknowledging the equipment. Manual small incision 
cataract surgery is more beneficial and fills the gap between high cost, high-tech phaco machines and conventional large-incision ECCE.,

In modern era of ophthalmology Phacoemulsification (PHACO) with foldable intraocular lens (IOL) is considered the best surgical technique for senile cataract. In spite of this manual small incision cataract surgery with rigid IOL is preferred for camp setups in developing countries because this type is less expensive and require no machinery. ${ }^{5}$

In modern days, best visual and refractive outcomes after surgery are given great importance. After surgery the action of differentiating to refractive surgery gets unimportant because every patient, without seeing the age demands best visual results and less dependence on spectacles. This is why the cataract surgery is now also considered a refractive surgery, not in the matter of clear lens extractions but also in standard procedures in all types of patients including adults. ${ }^{6}$

Intraocular lenses are designed to take place of the crystalline lens. Over the past decades, beyond adding spherical refractive power to the IOL, specific physiologic properties have also been included. One of such property is asphericity of the IOL design, this is to counterbalance the positive asphericity of prolate cornea, to give the same optical effects as the natural crystalline lens gives. The minimal aberrations improve the quality of the retinal image and enhance the visual quality with a spherical IOL.?

\section{Material and methods}

All subjects with senile mature cataract bilaterally age 40-65 years were included. Power of intraocular lens was between 16.0 dioptres (D) and 28.0 (D) implanted in posterior chamber after appropriate biometry. Distant best corrected visual outcomes with spheric and aspheric yellow tinted IOL were measured using standard Snellen chart. Quality of vision with both types of intraocular lenses were compared after one month of cataract (phacomulsification) surgery. Data was collected form Al-majeed laser eye hospital on basis of inclusion and exclusion criteria. Non-probability sampling technique was used to collect data. Both genders were included in study. Patients included in study were having senile mature cataract in both eyes with no posterior segment pathology with visual acuity less than $6 / 60$, equal to $6 / 60$ and 6/36 preoperatively measured with Snellen chart on basis of inclusion and exclusion criteria. Further, results were analyzed after SPSS version 21. Pre and post-operative visual acuity was measured by using standard snellen visual acuity testing chart. For the purpose of accuracy repetitive values were taken for each patient. Post-operative vision was recorded one month followed by phacoemulsification. Comparisons was made between spheric and asphereic acrylic yellow tinted intraocular lens by applying (descriptive) statistics and paired sample t- test for the analysis of results.

\section{Results}

Results of this study shows that pseduphakic patients with yellow tinted lens type aspheric hydrophilic implanted had recorded visual acuity of $6 / 9$ or more improved $6 / 6$. There were statistically significant differences in visual presentation. Post corrected visual acuity with spheric IOL total of 30 subjects 14 were fully corrected visual acuity 6/6, 12 patients with moderate visual acuity 6/9-6/12 and 4 patients with reduced 6/12-6/18 found respectively. Post op best corrected visual acuity with aspheric acrylic IOL total of 30 subjects 22 were fully corrected visual acuity $6 / 6$, while 8 patients with moderate visual acuity 6/6-6/9 found respectively. Pre -operative measurements shows that recorded visual acuity was less than $6 / 60$,equal to $6 / 60$ and $6 / 36$ of all subjects before implanting intra-ocular lenses. Age wise distribution was data shows quality of vision was more improved with age group 45-54 years as compared to 55-65 years since of age factor.

Descriptive statistics that with lens type spheric intraocular lens mean value .1867, standard deviation. 18889, t-value 2.517 and p-value 0.15 were calculated. While with Aspheric acrylic yellow tinted intraocular lens type mean, $\mathrm{SD}, \mathrm{t}$ and $\mathrm{p}$ value were found .800 . $.13493,2.5493$ and 0.15 respectively. The variance in values of mean, $\mathrm{SD}$ and $\mathrm{p}$ value with (95\% confidence interval) between two types of IOL were found 0.15 (Figure 1).

Figure 2 shows the post-operative comparison of quality of vision of spheric and aspheric yellow tinted intraocular lens. After phacomulsification data of patients with spheric intraoculation implantation out of 30 patients shows that 14 patients had fully corrected visual acuity 6/6, 12 patients moderate corrrected visual acuity 6/9-/612 while 4 patients visual acuity $6 / 18$ was recorded. While with lens type aspheric yellow tinted total of 30 patients 22 had best corrected visual acuity $6 / 6$ and 8 patients with $6 / 9-612$ was recorded. Results shows the variance in visual performance of two intraocular lenses implanted post-op of phacomulsification. Figure 3 shows the preoperative visual acuity of all subjects with senile cataract in both eyes which was recorde less than $6 / 60$, equal to $6 / 60$ and 6/36 respectively.

\begin{tabular}{|c|c|c|c|c|}
\hline Lens type & Mean & SD & $t$ & p-value \\
\hline $\begin{array}{l}\text { Spheric } \\
\text { intraocular lens }\end{array}$ & .1867 & .18889 & 2.517 & 0.15 \\
\hline $\begin{array}{l}\text { Aspheric acrylic } \\
\text { yellow tinted } \\
\text { intraocular lens }\end{array}$ & .800 & 13493 & 2.5493 & 0.15 \\
\hline
\end{tabular}

Figure I Descriptive statistics of spheric intraocular lens and aspheric acrylic yellow tinted intraocular lens. 


\section{Comparison of post -operative visual acuity of spheric IOL and aspheric acrylic yellow tinted IOL}

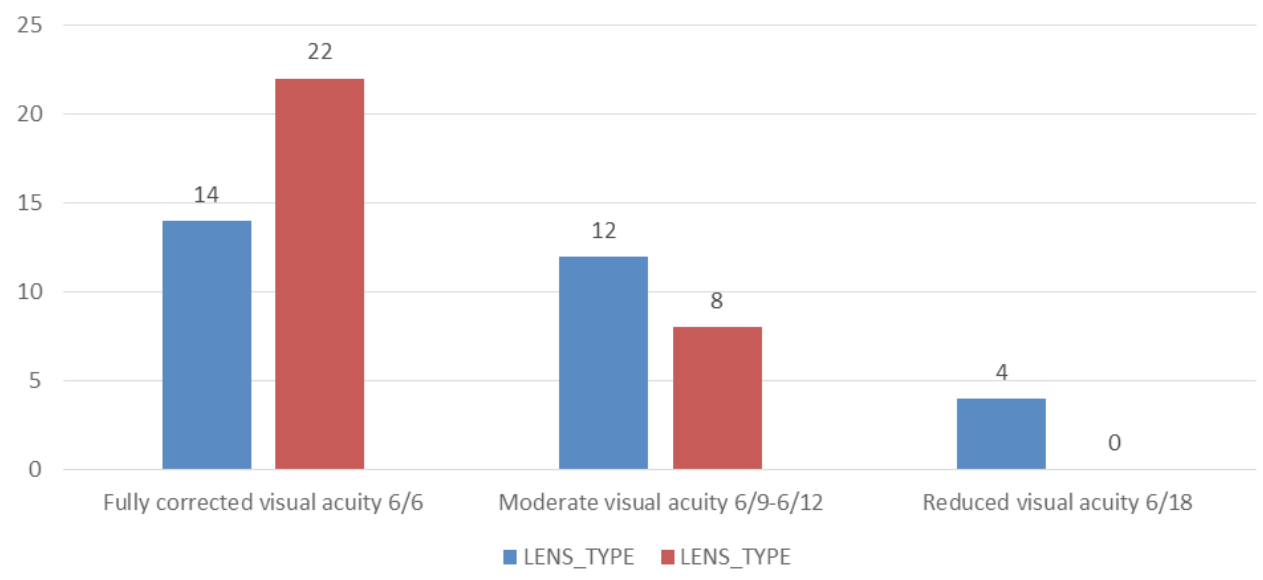

Figure 2 Comparison of post -op visual acuity of spheric intraocular lens and aspheric acrylic yellow tinted intraocular lens following cataract surgery.

Pre-operative visual acuity before implantation of spheric and aspheric IOL

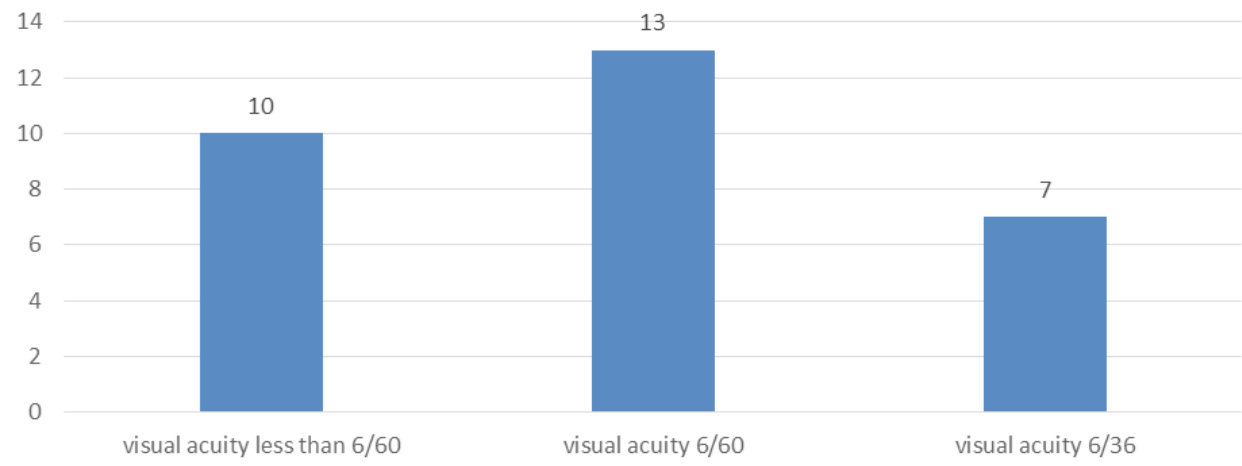

Chart Title

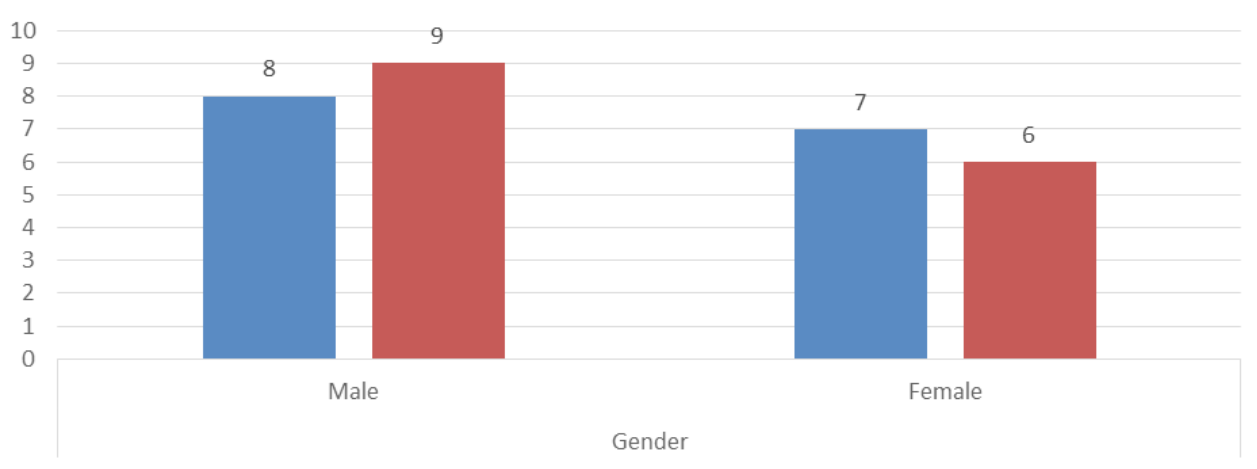

aspheric and aspheric yellow tinted IOL $\quad$ spheric and aspheric yellow tinted IOL

Figure 3 Comparison of pre -op visual acuity of spheric intraocular lens and aspheric acrylic yellow tinted intraocular lens.

\section{Discussion}

The study compared the quality of vision post phacoemulsification followed by Spheric and Aspheric hydrophilic acrylic yellow tinted intraocular lens in cataract patients. There found statistically significant difference between the two procedures. Post visual acuity was measured on standard Snellen acuity chart. Out of 60 subjects 30 were underwent phacoemulsification surgery following an aspheric hydrophilic acrylic IOL and 30 with spheric IOL. Mean \pm SD for spheric IOL and Aspheric IOL was $0.1867 \pm 0.1889$ and $0.800 \pm 0.1349$ respectively. The finding of this study showed that visual quality was better with Aspheric hydrophilic acrylic yellow tinted lens as $(\mathrm{p}<$ $0.05)$, statistically significant. 
Robert T et al., ${ }^{8}$ conducted a Randomized prospective longitudinal intrapatient comparison study between aspherical and spherical IOLs. Comparison of post operative visual quality was determined. Total 22 subjects were included who undergone bilateral phacoemulsification in cataract. Subjects were evaluated for their best corrected postoperative visual acuity with other additional parameters contrast sensitivity, spherical aberrations (SA), and higher order wave-front aberrations for a $4.5 \mathrm{~mm}$ and a $5.0 \mathrm{~mm}$ pupil were calculated after 3 months of surgery. Visual quality found better in the eyes implanted with aspheric IOLs than spheric. Moreover, spherical aberrations were significantly lower in eyes implanted with aspherical hydrophilic acrylic IOL. There found statistical difference between two groups $(P<0.001)$, in terms of quality of visual acuity. Study showed similar results to our study. However, they covered more variables in their study. ${ }^{8}$

In another study by Kim W et al., ${ }^{9}$ measurements were taken uniocularly for post-operative (phacoemulsification) patients with a spherical AcrySof MA30 IOL and aspheric acrylic Tecnis ZA9003 IOL. Study included 9 individuals with a spheric AcrySof IOL, 19 individuals with a spherical foldable IOL, and 24 individuals with an aspheric foldable IOL. Visual acuity component was measured by using LogMar notation. The myopic shift (myopic refractive error) and depth of focus were evaluated in that way. Results showed that patients with an aspheric foldable IOL had less spherical aberration and better vision quality as compared to others. Eyes with an aspheric IOL had a smaller myopic shift than eyes with other IOLs. Study mentioned similar results to our study outcomes, visual acuity and visual performance was better when fitted with and aspheric IOL. ${ }^{9}$

A Sandoval S et al., ${ }^{10}$ conducted a randomized study that included 54 eyes of 27 subjects who were Fitted IOLs following cataract surgery. Patients were divided into two sub groups on random sampling. Division was depending on the type of IOL implanted including spheric Acrys of and aspheric acrylic IOL. Visual acuity was measured repeatedly 1-3 months postoperatively through ETDRS test chart. Comparison between the two groups was evaluated and $\mathrm{P}<0.05$ was considered statistically significant. Preoperatively, statistically significant difference was observed in response to the subjective survey (near work, driving time, and far activities between two groups. Better vision quality and satisfaction were obtained by patients fitted with acrylic aspheric IOL than spheric Acrys of IOL. ${ }^{10}$

Yeni A conducted a retrospective study in a single centre on patients with cataract surgery following lens implantation. It was included medical records of the patients who were followed up for 6 months after surgery. Comparison of visual acuity was observed in that study between Natural Yellow Hydrophilic Aspheric IOL and spheric IOL. A total of 48 eyes of 48 patients, with their mean age of $66.84 \pm 9.72$ years ranging (43-88) years and equal distribution of both genders male and female were included. Mean of preoperative visual acuity for far was $0.81 \pm 0.30 \operatorname{LogMAR}$, and postoperatively mean was improved to $0.17 \pm 0.15$ LogMAR (standard visual acuity chart) at six months follow-up $(\mathrm{p}<0.05)$ in mean refractive spherical equivalent refractive error. Better visual performance and preferred refractive outcome as their spherical equivalent refractive error was found better with the implantation of Yellow tinted hydrophilic aspheric IOL as cpmared to spheric IOL. The Yellow Hydrophilic Aspheric IOL improved visual acuity outcomes in patients who underwent cataract surgery, without any problems, while protecting visual excellence. ${ }^{11}$

\section{Acknowledgments}

None.

\section{Conflicts of interest}

There are no financial conflicts of interest.

\section{Funding}

None.

\section{References}

1. U Dillinger $P$, Anterist N. Impact of a modified optic design on visual function: clinical comparative study. $J$ Cataract Refract Surg. 2003;29:652-660.

2. Hennig A, Puri LR, Sharma H, et al. Foldable vs rigid lenses after phacoemulsification for cataract surgery: a randomized controlled trial. Eye. 2014;28(5):pp.567-575.

3. Devendra J, Agarwal S, Singh PK. A comparative study of clear corneal phacoemulsification with rigid IOL versus SICS; the preferred surgical technique in low socio-economic group patients of rural areas. Journal of clinical and diagnostic research. 2014;8(11):VC01.

4. Ang M, Evans JR, Mehta JS. Manual small incision cataract surgery (MSICS) with posterior chamber intraocular lens versus extracapsular cataract extraction (ECCE) with posterior chamber intraocular lens for age-related cataract. Cochrane Database of Systematic Reviews. 2014;(11).

5. Venkatesh Rengaraj, Tan Colin SH. Phacoemulsification versus manual small-incision cataract surgery for white cataract. Journal of Cataract \& Refractive Surgery. 2010;36(11):1849-1854.

6. Borkenstein AF, Borkenstein EM. Long-term clinical results and scanning electron microscopic analysis of the aspheric, hydrophobic, acrylic intraocular lens CT LUCIA 611P (Y). Clinical Ophthalmology (Auckland, NZ). 2018;12:1219.

7. Schuster AK, Tesarz J, Vossmerbaeumer U. The impact on vision of aspheric to spherical monofocal intraocular lenses in cataract surgery:a systematic review with meta-analysis. Ophthalmology. 2013;120(11):2166-2175.

8. Robert T Ang, Gladness A, Jesse B Caguioa, et al. Comparison in the quality of vision and spherical aberration between spherical and aspheric intraocular lenses. J Refract Surg. 2014;33(1).

9. Kim W, Nomdo M, Steven A, et al. Comparison of Optical Performance in Eyes Implanted with Aspheric Foldable, Spherical Foldable, and Rigid PMMA IOLs. PMID. 2011;27(2):98-105.

10. Sandoval S, Fernández de C, Vroman D, et al. Comparison of visual outcomes, photopic contrast sensitivity, wavefront analysis, and patient satisfaction following cataract extraction and IOL implantation:aspheric vs. spherical acrylic lenses. Eye (Lond). 2008;22(12):1469-1475.

11. Yiğit U, Çavuşoğlu E, Onur İU, et al. Clinical Outcomes Following Implantation of A New Aspheric Monofocal Intraocular Lens. JAREM. 2019;9(Supplement 1):S35-40. 